\title{
MULTIPOINT INITIAL-FINAL VALUE PROBLEM FOR THE MODEL OF DEVIS WITH ADDITIVE WHITE NOISE
}

A.S. Konkina, South Ural State University, Chelyabinsk, Russian Federation, konkinaas@susu.ru

The evolution of the free surface of the filtering fluid in a reservoir of limited power is modeled by the Davis equation with homogeneous Dirichlet conditions. Depending on the nature of the free term describing the internal source of the liquid, the model will be deterministic or stochastic. The deterministic model has been studied in various aspects by many researchers with different initial (initial-final value conditions). The stochastic model is studied for the first time. The main result is the proof of the unique solvability of the evolutionary model with an additive white noise and a multipoint initial-final value condition.

Keywords: white noise; Wiener K-process; Davis model; multipoint initial-final value problem; stochastic model.

Introduction. Let $\Omega \subset \mathbb{R}^{n}$ be a bounded domain with boundary $\partial \Omega$ of class $C^{\infty}$. In the cylinder $\Omega \times \mathbb{R}_{+}$consider the evolutionary Davis model

$$
\begin{gathered}
(\lambda-\Delta) u_{t}=\alpha \Delta u-\beta \Delta^{2} u+f, \\
u(x, t)=\Delta u(x, t)=0, \quad(x, t) \in \partial \Omega \times \mathbb{R}_{+},
\end{gathered}
$$

where $\lambda \in \mathbb{R}, \alpha, \beta \in \mathbb{R}_{+}$characterize the environment. Equation (1), together with conditions (2), where the free term $f=f(t)$ corresponds to the deterministic source of the liquid, can be reduced to an evolution Sobolev type equation

$$
L \dot{u}=M u+f
$$

where the operators $L \in \mathcal{L}(\mathfrak{U} ; \mathfrak{F})$ and $M \in \mathcal{C} l(\mathfrak{U} ; \mathfrak{F})$ act in some Banach spaces $\mathfrak{U}$ and $\mathfrak{F}$. The same problem (1), (2), where the external action $f=f(t)$ is a white noise leads to a stochastic Sobolev type equation

$$
L d u=M u d t+N d W .
$$

Here $\mathfrak{U}$ is a Banach space, $\mathfrak{F}$ is a real separable Hilbert space, the operators $L \in \mathcal{L}(\mathfrak{U} ; \mathfrak{F})$, $M \in \mathcal{C l}(\mathfrak{U} ; \mathfrak{F})$, and $W=W(t)$ is an $\mathfrak{F}$-valued Wiener $K$-process.

Take $\tau_{0}=0$ and $\tau_{j} \in \mathbb{R}_{+}$, such that $\tau_{j-1}<\tau_{j}$ for $j=\overline{1, n}$. Equations (3), (4) can be supplemented by a multipoint initial-final value condition

$$
\lim _{t \rightarrow \tau_{0}+} P_{0}\left(u(t)-\xi_{0}\right)=0, P_{j}\left(u\left(\tau_{j}\right)-\xi_{j}\right)=0, j=\overline{1, n} .
$$

where $P_{j}$ are relatively spectral projectors [1].

At the end of the statement of the problem we note that three directions of the solution of the problem (4), (5) are actively developing now. White noise is used to solve the problem, which is understood as the generalized derivative of the Wiener $K$-process [2]. We also note the approach presented by the school of I.V. Melnikova, in which equation 
(4) is considered in Schwartz spaces, where the generalized derivative of a Wiener $K$ process makes sense [3]. Meanwhile, a new approach is actively developing in the studies of equation (4), where by "white noise" is meant the Nelson - Glickich derivative of the Wiener $K$-process [4].

1. A Determinate Sobolev Type Equation. Let $\mathfrak{U}$ and $\mathfrak{F}$ be Banach spaces, operators $L \in \mathcal{L}(\mathfrak{U} ; \mathfrak{F})$ (that is, linear and continuous) and $M \in \mathcal{C l}(\mathfrak{U} ; \mathfrak{F})$ (i.e., linear, closed, and densely defined). Let $M$ be $(L, p)$-sectorial, $p \in\{0\} \cup \mathbb{N}$. Consider the Sobolev type equation (3) equipped with condition

$$
\lim _{t \rightarrow \tau_{0}+} P_{0}\left(u(t)-u_{0}\right)=0, P_{j}\left(u\left(\tau_{j}\right)-u_{j}\right)=0, j=\overline{1, n} .
$$

Moreover, we recall that the condition of an $(L, p)$-sectoriality of the operator $M$ is a sufficient condition for the existence of degenerate analytic semigroups of operators [5]

$$
U^{t}=\frac{1}{2 \pi i} \int_{\Gamma}(\mu L-M)^{-1} L e^{\mu t} d \mu \quad \text { и } \quad F^{t}=\frac{1}{2 \pi i} \int_{\Gamma} L(\mu L-M)^{-1} e^{\mu t} d \mu,
$$

defined on spaces $\mathfrak{U}$ and $\mathfrak{F}$ respectively. Introduce the kernels $\operatorname{ker} U^{\cdot}=\mathfrak{U}^{0}$, ker $F^{\cdot}=\mathfrak{F}^{0}$ and the images $\operatorname{im} U^{\cdot}=\mathfrak{U}^{1}, \operatorname{im} F^{\cdot}=\mathfrak{F}^{1}$ of these semigroups. Suppose that condition

$$
\mathfrak{U}^{0} \oplus \mathfrak{U}^{1}=\mathfrak{U} \quad\left(\mathfrak{F}^{0} \oplus \mathfrak{F}^{1}=\mathfrak{F}\right),
$$

is fulfilled. It holds either in the case of strong $(L, p)$-sectorial operator $M$ on the right (left), $p \in\{0\} \cup \mathbb{N}$ or the reflexivity of space $\mathfrak{U}(\mathfrak{F})$.

Denote by $L_{k}\left(M_{k}\right)$ contraction of operator $L(M)$ on $\mathfrak{U}^{k}\left(\operatorname{dom} M \cap \mathfrak{U}^{k}\right), k=0,1$. If the operator $M$ is $(L, p)$-sectorial $p \in\{0\} \cup \mathbb{N}$, then $L_{k} \in \mathcal{L}\left(\mathfrak{U}^{k} ; \mathfrak{F}^{k}\right), M_{k} \in \mathcal{C} l\left(\mathfrak{U}^{k} ; \mathfrak{F}^{k}\right), k=0,1$, and there exists an operator $M_{0}^{-1} \in \mathcal{L}\left(\mathfrak{F}^{0} ; \mathfrak{U}^{0}\right)$, as well as a projector $P=s-\lim _{t \rightarrow 0+} U^{t}$, $\left(Q=s-\lim _{t \rightarrow 0+} F^{t}\right)$, splitting space $\mathfrak{U}(\mathfrak{F})$ according to $(\mathrm{A} 1)$, and $\mathfrak{U}^{1}=\operatorname{im} P\left(\mathfrak{F}^{1}=\operatorname{im} Q\right)$. Introduce one more condition:

$$
\text { there exists an operator } \quad L_{1}^{-1} \in \mathcal{L}\left(\mathfrak{F}^{1} ; \mathfrak{U}^{1}\right)
$$

which occurs in the case of a strong $(L, p)$-sectoriality of the operator $M, p \in\{0\} \cup \mathbb{N}$. It was shown earlier that (A1) together with the condition of $(L, p)$-sectoriality of the operator $M, p \in\{0\} \cup \mathbb{N}$, gives a strong $(L, p)$-sectoriality of the operator $M$ on the right (left), $p \in\{0\} \cup \mathbb{N}$, and if we add condition (A2) to them, we get a strong ( $L, p)$-sectoriality of the operator $M, p \in\{0\} \cup \mathbb{N}[5]$. Then the operator $G=M_{0}^{-1} L_{0} \in \mathcal{L}\left(\mathfrak{U}^{0}\right)$ is nilpotent of degree $p$, and the operator $S=L_{1}^{-1} M_{1} \in \mathcal{C l}\left(\mathfrak{U}^{1}\right)$ is sectorial.

Finally, introduce one more important condition on the relative spectrum of an operator $M[6]$ :

$$
\left.\begin{array}{c}
\sigma^{L}(M)=\bigcup_{j=0}^{n} \sigma_{j}^{L}(M), n \in \mathbb{N} \text {, with } \sigma_{j}^{L}(M) \neq \emptyset \text { contained in a bounded } \\
\text { domain } D_{j} \subset \mathbb{C} \text { with a piecewise smooth boundary } \partial D_{j}=\Gamma_{j} \subset \mathbb{C} . \\
\text { Moreover } \overline{D_{j}} \cap \sigma_{0}^{L}(M)=\emptyset \text { and } \overline{D_{k}} \cap \overline{D_{l}}=\emptyset \text { for all } j, k, l=\overline{1, n}, k \neq l .
\end{array}\right\}
$$


Construct relatively spectral projectors $[6] P_{j} \in \mathcal{L}(\mathfrak{U})$ and $Q_{j} \in \mathcal{L}(\mathfrak{F}), j=\overline{1, n}$, of the form

$$
P_{j}=\frac{1}{2 \pi i} \int_{\Gamma_{j}}(\mu L-M)^{-1} L d \mu, Q_{j}=\frac{1}{2 \pi i} \int_{\Gamma_{j}} L(\mu L-M)^{-1} d \mu, j=\overline{1, n} .
$$

It turns out that under the condition of $(L, p)$-sectoriality of the operator $M$ and conditions (A1), (A2), $P_{j} P=P P_{j}=P_{j}$ and $Q_{j} Q=Q Q_{j}=Q_{j}, j=\overline{1, n}$. Hence, in this case there exists a projector $P_{0}=P-\sum_{j=1}^{n} P_{j}, P_{0} \in \mathcal{L}(\mathfrak{U})$.

Suppose that conditions (A1) - (A3) are satisfied. Fix $\tau_{0}=0, \tau_{j} \in \mathbb{R}_{+}\left(\tau_{j-1}<\tau_{j}\right)$, $u_{j} \in \mathfrak{U}, j=\overline{0, n}$, and consider the multipoint initial-final value condition (6) for a linear Sobolev type equation (3). A vector function $u \in C^{1}\left(\left[\tau_{0}, \tau_{n}\right] ; \mathfrak{U}\right) \cap C\left(\left[\tau_{0}, \tau_{n}\right] ; \mathfrak{U}\right)$, satisfying (3), is called a solution of (3); solution $u=u(t)$ of equation (3) satisfying (6) is called $a$ solution of a multipoint initial-final value problem (3), (6).

Theorem 1. [6] Let the operator $M$ be $(L, p)$-sectorial, (A1) - (A3) hold. Then for any vector-function $f^{0} \in C^{p}\left(\left[\tau_{0}, \tau_{n}\right] ; \mathfrak{F}^{0}\right) \cap C^{p+1}\left(\left(\tau_{0}, \tau_{n}\right) ; \mathfrak{F}^{0}\right), f^{1} \in C\left(\left[\tau_{0}, \tau_{n}\right] ; \mathfrak{F}^{1}\right)$ there exists a unique solution of (3), (6), given by

$$
u(t)=-\sum_{q=1}^{p} G^{q} M_{0}^{-1} f^{0(q)}(t)+\sum_{j=1}^{n}\left(U_{j}^{t-\tau_{j}} u_{j}+\int_{\tau_{j}}^{t} U_{j}^{t-s} L_{1 j}^{-1} Q_{j} f(s) d s\right) .
$$

2. The Spaces of Noises. Let $\Omega \equiv(\Omega, \mathcal{A}, \mathbf{P})$ be complete probability space and let $\mathbb{R}$ be the set of real nimbers endowed Borel $\sigma$-algebra. A measurable mapping $\xi: \Omega \rightarrow \mathbb{R}$ is called a random variable. Note that all random variables, having a normal distribution (i.e. gaussian), are contained in space $\boldsymbol{L}_{2}$ see [4].

Consider two mappings $f: \mathfrak{I} \rightarrow \boldsymbol{L}_{\mathscr{2}}$, matching each $t \in \mathfrak{I}$ to a random variable $\xi \in \boldsymbol{L}_{\mathscr{2}}$, and $g: \boldsymbol{L}_{\boldsymbol{2}} \times \Omega \rightarrow \mathbb{R}$, matching each pair $(\xi, \omega)$ to a point $\xi(\omega) \in \mathbb{R}$, where $\mathfrak{I} \subset \mathbb{R}$ is some interval. By a stochastic process we call a mapping $\eta: \mathfrak{I} \times \Omega \rightarrow \mathbb{R}$, having the form $\eta=\eta(t, \omega)=g(f(t), \omega)$. Note that the stochastic process $\eta=\eta(t, \cdot)$, if $t \in \mathfrak{I}$ is fixed, is a random variable, and the stochastic process $\eta=\eta(\cdot, \omega)$, if $\omega \in \Omega$ is fixed, will be called(sample) trajectory. A stochastic process $\eta$ is called continuous, if for almost all $\omega \in \Omega$ a trajectory $\eta(t, \omega)$ is continuous on $\mathfrak{I}$.

Denote by $\mathcal{P} \equiv \mathcal{P}(\mathfrak{I} \times \Omega ; \mathfrak{U})$ a space of stochastic processes. The space of continuous stochastic processes whose random variables belong to $\boldsymbol{L}_{\boldsymbol{Z}}$ is denoted by $\boldsymbol{C} \boldsymbol{L}_{\boldsymbol{Z}}$, i.e. $\eta \in \boldsymbol{C} \boldsymbol{L}_{\boldsymbol{Z}}$, if $\eta(t, \cdot) \in \boldsymbol{L}_{\boldsymbol{2}}$ for all $t \in \mathfrak{I}$. Note that $\boldsymbol{C} \boldsymbol{L}_{2}$ is a subspace of $\mathcal{P}$. Note that the space $\boldsymbol{C L}_{\boldsymbol{2}}$ in particular contains those stochastic processes, whose all trajectories are continuous, and all (independent) random variables are Gaussian.

Consider the operator $K \in \mathcal{L}(\mathbb{R})$, whose spectrum $\sigma(K)$ is positive, i.e. $\sigma(K) \in \mathbb{R}_{+}$. This is possible when $K$ positive and self-adjoint. The sequence of eigenvalues of the operator $K$ is denoted by $\left\{\lambda_{k}\right\}$. Suppose that the spectrum $\sigma(K)$ is discrete, finite and is condensed only to the zero point. Enumerate in $\left\{\lambda_{k}\right\}$ nonincreasing order, taking into account their multiplicities.

Operator $K$ is called nuclear, if $\operatorname{Tr} K=\sum_{k=1}^{\infty} \lambda_{k}<+\infty$. We note that the linear span of the set $\left\{\varphi_{k}\right\}$ of the corresponding eigenfunctions of the operator $K$ is dense in $\mathbb{R}$. Let us define the Brownian motions, take the sequence $\left\{\xi_{k}\right\}$, of independent random variables. 
Definition 1. A stochastic process

$$
\beta(t) \equiv \beta(t, \omega)=\sum_{k=0}^{\infty} \xi_{k} \sin \frac{\pi}{2}(2 k+1), t \in \overline{\mathbb{R}}_{+},
$$

is called a Brownian motion, if

(W1) $\beta(0)=0$ almost everywhere in $\Omega$, and the trajectories of stochastic process $\beta(t)$ are almost surely continuous on $\overline{\mathbb{R}}_{+}$;

(W2) the trajectories of stochastic process $\beta(t)$ are almost surely undifferentiable at any point $t \in \overline{\mathbb{R}}_{+}$and on any interval $\mathfrak{I} \subset \overline{\mathbb{R}_{+}}$have unbounded variation.

As an example consider the Wiener $K$-process [4]

$$
W(t)=\sum_{k=0}^{\infty} \sqrt{\lambda_{k}} \beta_{k}(t) \varphi_{k}
$$

where $\beta_{k}(t)$ are Brownian motions, $k \in \mathbb{N}$.

Theorem 2. [2] For any nuclear operator $K \in \mathcal{L}(\mathfrak{U})$ and arbitrary sequence of Brownian motions $\left\{\xi_{j}\right\}$ a Wiener $K$-process $W \in \boldsymbol{C L}_{2}$.

For the solvability of problem (4), (5) we need another condition

$$
Q N=N \text {. }
$$

Then the formal solution $u=u(t)$ of the multipoint initial-final problem (5) for equation (4) has the form

$$
u(t)=U_{0}^{t} \xi_{0}+\sum_{j=1}^{m}\left[\int_{\tau_{j}}^{t} U^{t-s} L_{1 j}^{-1} Q_{j} N d W(s)+U^{t-\tau_{j}} \xi_{j}+L_{1 j}^{-1} Q_{j} N W(t)\right] .
$$

Theorem 3. Let the operator $M$ be (L,p)-sectorial and conditions (A1)-(A4) be fulfilled. Then for arbitrary $\mathfrak{U}^{1}$-valued Gaussian random variables $\xi_{j}, j=\overline{0, n}$ independent on $W(t)$ and satisfying (9), there exists a unique strong solution of (4), (5), given by (10).

3. The Davis Model. Consider problem (1), (2), where white noise appears as an external action of $f=f(t)$. Let $\mathfrak{F}=L_{2}(\Omega)$ and $\mathfrak{U}=\left\{u \in W_{2}^{2}(\Omega): u(x)=0, x \in \partial \Omega\right\}$. Operators $L$ and $M$ are given by formulas $L=\lambda-\Delta$ and $M=\alpha \Delta-\beta \Delta^{2}$,

$$
\operatorname{dom} M=\mathfrak{U} \cap\left\{u \in W_{2}^{4}(\Omega): \Delta u(x)=0, x \in \partial \Omega\right\} .
$$

Obviously, for all $\lambda \in \mathbb{R}$ operator $L \in \mathcal{L}(\mathfrak{U} ; \mathfrak{F})$, and for all $\alpha \in \mathbb{R}, \beta \in \mathbb{R} \backslash\{0\}$ operator $M \in \mathcal{C l}(\mathfrak{U} ; \mathfrak{F})$.

Lemma 1. For all $\lambda \in \mathbb{R}, \alpha, \beta \in \mathbb{R}_{+}$operator $M$ is $(L, 0)$-sectorial.

Let $\left\{\varphi_{k}\right\}$ be an orthonormal set of eigenfunctions of the homogeneous Dirichlet problem for the Laplace operator $\Delta$ in a domain $\Omega$, numbered in the nonincreas in a order of the eigenvalues $\left\{\lambda_{k}\right\}$ taking into account their multiplicities. Since

$$
(\mu L-M) u=\sum_{k=1}^{\infty}\left(\mu \lambda-(\mu+\alpha) \lambda_{k}+\beta \lambda_{k}^{2}\right)<u, \varphi_{k}>\varphi_{k}
$$


for any $u \in \operatorname{dom} M, \mu \in \mathbb{C}$, then

$$
(\mu L-M)^{-1}=\sum_{k=1}^{\infty} \frac{<\cdot, \varphi_{k}>}{\beta \lambda_{k}^{2}-\alpha \lambda_{k}+\mu\left(\lambda-\lambda_{k}\right)} \varphi_{k}
$$

The series in (11) converges absolutely and uniformly on any compact set in $\mathbb{C}$, free of points

$$
\mu_{k}=\lambda_{k} \frac{\beta \lambda_{k}-\alpha}{\lambda_{k}-\lambda}, k \in \mathbb{N} .
$$

Since the spectrum $\sigma(\Delta)$ negative, discrete, finite and condensed only to $-\infty$, then it follows from (12) that the $L$-spectrum $\sigma^{L}(M)$ operator $M$ it real, discrete, finite and condensed only to $-\infty$. In considering the problem, we confine ourselves to the values of the parameter $\lambda$ lying in the spectrum of the operator $\Delta$. Therefore, from the set of numbers (12) it is necessary to delete the numbers $\mu_{k}$ with numbers $k$ for which $\lambda=\lambda_{k}$.

So, let $\lambda \in \sigma(\Delta)$, then we get

$$
\begin{gathered}
(\mu L-M)^{-1}=\sum_{k=1}^{\infty}{ }^{\prime} \frac{<\cdot, \varphi_{k}>\varphi_{k}}{\beta \lambda_{k}^{2}-\alpha \lambda_{k}+\mu\left(\lambda-\lambda_{k}\right)}+\sum_{\lambda_{k=\lambda}} \frac{<\cdot, \varphi_{k}>\varphi_{k}}{\beta \lambda^{2}-\alpha \lambda}, \\
R_{\mu}^{L}(M)=\sum_{k=1}^{\infty} '\left(\mu+\lambda_{k} \frac{\beta \lambda_{k}-\alpha}{\lambda-\lambda_{k}}\right)^{-1}<\cdot, \varphi_{k}>\varphi_{k}=L_{\mu}^{L}(M), \\
(\nu L-M)^{-1} L_{\mu}^{L}(M)=\sum_{k=1}^{\infty} '\left(\mu+\lambda_{k} \frac{\beta \lambda_{k}-\alpha}{\lambda-\lambda_{k}}\right)^{-1} \times \frac{<\cdot, \varphi_{k}>\varphi_{k}}{\beta \lambda_{k}^{2}-\alpha \lambda_{k}+\mu\left(\lambda-\lambda_{k}\right)},
\end{gathered}
$$

where the prime at the sum sign means the absence of summands with numbers $k$ such that $\lambda=\lambda_{k}$. Hence it is not difficult to obtain the strong $L$-spectoriality of the operator $M$. For the sake of simplicity, we take the operator $N=Q$, then condition (9) is obviously satisfied. Denote by $\left\{\mu_{k}\right\}$ a sequence of eigenvalues of the Laplace operator $\Delta$ with condition (2) in a domain $\Omega$, numbered in nonincreasing order with taking into account their multiplicities, and by $\left\{\varphi_{k}\right\}$ a sequence of eigenfunctions. Then

$$
u(t)=\sum_{j=1}^{n} \sum_{k=1}^{\infty}{ }^{\prime} e^{\nu_{k} t}\left\langle\xi_{j}, \varphi_{k}\right\rangle \varphi_{k}+\sum_{j: \nu_{j} \in \sigma_{j}^{L}(M)} \sum_{k=1}^{\infty}{ }^{\prime} \frac{\sqrt{\lambda_{k}}}{\lambda-\mu_{k}} \int_{\tau_{j}}^{t} e^{\nu_{k}(t-s)} d \beta_{k}(s) \varphi_{k},
$$

where $\nu_{k}=\frac{\left(\alpha \mu_{k}-\beta \mu_{k}^{2}\right)}{\left(\alpha-\mu_{k}\right)}$ are the points of the $L$-spectrum of the operator $M,\left\{\lambda_{k}\right\}$ are the eigenvalues of a special constructed nuclear operator $K$. A prime at a sum sign means the absence of terms such that $\lambda=\mu_{k}$.

Corollary 1. Let $\lambda \in \mathbb{R}, \alpha, \beta \in \mathbb{R}_{+}$, and conditions (A1) - (A4) be fulfilled. Let $\xi_{j}$ satisfy (9). Then there exists a unique strong solution of (1), (2), (5), given by (13).

Acknowledgements. The work was supported by Act 211 Government of the Russian Federation, contract No. 02.A03.21.0011. 


\section{References}

1. Zagrebina S.A., Konkina A.S. The Multipoint Initial-Final Value Condition for the Navier - Stokes Linear Model. Bulletin of the South Ural State University. Series: Mathematical Modelling, Programming and Computer Software, 2015, vol. 8, no. 1, pp. 132-136. DOI: $10.14529 / \mathrm{mmp} 150111$

2. Zagrebina S.A., Soldatova E.A., Sviridyuk G.A. The Stochastic Linear Oskolkov Model of the Oil Transportation by the Pipeline. Semigroups of Operators - Theory and Applications, Bedlewo, Poland, Springer, 2013, pp. 317-325. DOI:10.1007/978-3-319-12145-1-20

3. Melnikova I.V., Filinkov A.I., Alshansky M.A. Abstract Stochastic Equations II. Solutions in Spaces of Abstract Stochastic Distributions. Journal of Mathematical Sciences, 2003, vol. 116, no. 5, pp. 3620-3656. DOI: 10.1023/A:1024159908410

4. Favini A., Sviridyuk G.A., Manakova N.A. Linear Sobolev Type Equations with Relatively p-Sectorial Operators in Space of "Noises". Abstract and Applied Analysis, 2015, vol. 2015, Article ID 697410. 8 p. DOI: $10.1155 / 2015 / 697410$

5. Sviridyuk G.A., Fedorov V.E. Linear Sobolev Type Equations and Degenerate Semigroups of Operators. Utrecht, Boston, Köln, VSP, 2003. DOI: 10.1515/9783110915501

6. Zagrebina S.A. Multipoint Initial-Final Value Problem for the Linear Model of Plane-Parallel Thermal Convection in Viscoelastic Incompressible Fluid. Bulletin of the South Ural State University. Series: Mathematical Modelling, Programming and Computer Software, 2014, vol. 7 , no. 3, pp. 5-22. DOI: $10.14529 / \mathrm{mmp} 140301$

Received March 25, 2017

УДК 517.9

DOI: $10.14529 / \mathrm{mmp} 170212$

\section{МНОГОТОЧЕЧНАЯ НАЧАЛЬНО-КОНЕЧНАЯ ЗАДАЧА ДЛЯ МОДЕЛИ ДЕВИСА С АДДИТИВНЫМ БЕЛЫМ ШУМОМ}

A.C. Конкина, Южно-Уральский государственный университет, г. Челябинск

Эволюция свободной поверхности фильтрующейся жидкости в пласте ограниченной мощности моделируется уравнением Девиса с однородными условиями Дирихле. В зависимости от характера свободного члена,описывающего внутренний источник жидкости, модель будет детерминированной или стохастической. Детерминированная модель изучалась в разных аспектах многими исследователями с различными начальными (начально-конечными) условиями. Стохастическая модель изучается впервые. Основной результат-докозательство однозначной разрешимости эволюционной модели с аддитивным белым шумом и многоточечным начально-конечным условием.

Ключевые слова: белый шум; винеровский К-процесс; модель Девиса; многоточечная начально-конечная задача; стохастическая модель.

Александра Сергеевна Конкина, ассистент кафедры «Уравнения математической физики», аспирант кафедры «Математическое и компьютерное моделирование», Южно-Уральский государственный университет (г. Челябинск, Российская Федерация), konkinaas@susu.ru.

Поступила в редакиию 25 марта 2017 г. 